\title{
Analyzing the microstructure and related properties of 2D materials by transmission electron microscopy
}

\author{
Yun-Yeong Chang, Heung Nam Han and Miyoung Kim ${ }^{*}$ (D)
}

\begin{abstract}
Two-dimensional materials such as transition metal dichalcogenide and graphene are of great interest due to their intriguing electronic and optical properties such as metal-insulator transition based on structural variation. Accordingly, detailed analyses of structural tunability with transmission electron microscopy have become increasingly important for understanding atomic configurations. This review presents a few analyses that can be applied to two-dimensional materials using transmission electron microscopy.
\end{abstract}

Keywords: Transmission electron microscopy, 2D materials, Transition metal dichalcogenide, Graphene, Van der Waals heterostructure

\section{Introduction}

Two-dimensional materials are considered as new candidates for future electronic and optical low-dimensional materials. In particular, the most spotlighted materials are graphene and transition metal dichalcogenides (TMD). TMDs are materials of type $\mathrm{MX}_{2}$, where $\mathrm{M}$ represents transition metal atoms such as Mo and W and $\mathrm{X}$ represents chalcogen atoms such as $\mathrm{S}$, Se, and others. In contrast to graphene, TMDs have band gaps (Manzeli et al. 2017). The properties of graphene and TMDs' can be tuned using varying growth conditions. Especially for TMDs, the band gap can be modified by decreasing the thickness from a few layers to a monolayer, by creating defects or implantations on the pristine surface and inducing electron-beam irradiation on the surface. For example, the most investigated material, $\mathrm{MoS}_{2}$, shows an indirect band gap in its bulk state, but when the layers are decreased into monolayers, the band gap changes into a direct band gap (He et al. 2014). This leads to better photoluminescence efficiency ( $\mathrm{He}$ et al. 2014; Wang et al. 2015).

Both graphene and TMDs form van der Waals bonds, which is a weak force of attraction between the layers when they are stacked into few layers. In bilayer $2 \mathrm{D}$

\footnotetext{
* Correspondence: mkim@snu.ac.kr

Department of Materials Science and Engineering, Seoul National University, Seoul 151-744, Korea
}

materials, the stacking sequence modifies the crystal symmetry and equilibrium distance, which affects the physical properties of $2 \mathrm{D}$ materials, such as band gap, phonon vibration frequency, and superconductivity (He et al. 2014; Yan et al. 2015). Recent studies on the manipulation of the interlayer sequence twist angle showed electron tunneling in the heterostructure of 2D materials (Ribeiro-Palau et al. 2018). For example, graphene showed a strong coupling effect when the bilayers were twisted at the angle of $1.1^{\circ}$, which is called the magic angle (Cao et al. 2018). Likewise, the TMD heterostructure demonstrates angle sensitivity in the formation of interlayer excitons (Rivera et al. 2015; Ribeiro-Palau et al. 2018).

Other 2D materials such as $1 \mathrm{~T}-\mathrm{TaSe}_{2}$ and $\mathrm{TaS}_{2}$ have a unique characteristic imparted by Fermi nesting, the charge density wave (CDW) (Johannes and Mazin 2008). The CDW is the periodic modulation of the charge density related to the periodic lattice distortion, which is observed as periodic structural changes in the atomic sites (Hossain et al. 2017). This lattice distortion occurs in two different forms, i.e., the commensurate and incommensurate structures; commensurate structures have electron density in the rational multiple of lattice distortion, whereas incommensurate structures have periodicities in the irrational number (Chen et al. 2016). At low temperatures, the property of CDW presents the 
superconductivity when it orders. Because of such distinct characteristics of CDW, much effort has been made to elucidate the underlying mechanism (Castro Neto 2001; Calandra 2015).

To understand the characteristics described, information on the atomic configuration of $2 \mathrm{D}$ materials is essential. Transmission electron microscopy (TEM) is the most commonly used technique for obtaining structural information. In particular, the $\mathrm{Z}$ contrast nature of high-angle annular dark-field (HAADF) scanning transmission electron microscopy (STEM) imaging enables to discern each atom in the 2D layer. The well-known disadvantages of TEM, such as knock-on damage and resolution problems, have been overcome with the recent progress in aberration correction. Aberration-corrected TEM enables to image atomic configurations of 2D layered structures at low acceleration voltages, which significantly reduces knock-on damage. Moreover, the domain patterns that form between the van der Waals layers, such as moiré patterns, can be identified with TEM, and the CDW changes can also be depicted in the diffraction patterns.

This review presents the TEM observation of defects and implantation and structural changes of 2D layers. In addition, research regarding the microstructure of $2 \mathrm{D}$ materials with atomic resolution imaging and electrical property measurements is described.

\section{Observation of defects, interstitial sites, and single atoms in 2D materials}

TEM has long been used to observe individual atoms, defects, and interstitial sites. Atomic-resolution information on the crystal structure of 2D materials by TEM has gained immense importance because the band structure of the material significantly changes in response to the defects, number of layers, and atomic configurations (Wang et al. 2012; Wang et al. 2017; Elibol et al. 2018). For example, $\mathrm{MoS}_{2}$ ferromagnetism is enhanced with the fabrication of atomic vacancies with electrons or ion beam. In addition, semiconducting $\mathrm{MoTe}_{2}$ showed a new mid-gap state in the band gap induced by imbedded quantum dots and quantum walls (Lin et al. 2016; Elibol et al. 2018). The defects also affect the localization of spin-orbit coupling in graphene. The coupling effect modifies the spin transport characteristics of TMD and graphene layers (Garcia et al. 2018). Furthermore, in specific graphene edge structures, a new band gap and spin state are induced when an electric field is applied (Wang et al. 2017). Because of the tunability of the electrical property with structure, the fabrication of materials with defects, interstitial sites, vacancies, and edge construction has become important.

The accurate identification of atomic configurations of $2 \mathrm{D}$ materials is the main issue in material tuning.
Thus, effort has been made to clearly analyze the point or line defects in structures by using TEM. However, radiation-induced damage remains one of the main limitations of high-resolution transmission electron microscopy (HRTEM) of 2D materials (Meyer et al. 2012). To solve this problem, Garcia et al. (2018) investigated the e-beam irradiation effects on the $\mathrm{MoS}_{2}$ layer at different $\mathrm{kV}$ levels. They calculated the energy transfer from the electron beam to the material and compared it with the displacement threshold energy of Mo and S. At $200 \mathrm{kV}$ and $120 \mathrm{kV}$, structural damage was observed, whereas the sample was stable at $80 \mathrm{kV}$. This result indicates that an electron beam of $80 \mathrm{kV}$ is optimum for reducing structural damage to $\mathrm{MoS}_{2}$. This acted as the basis for the TEM analysis of the possible stable state. The difficulty of immaculate sample preparation is another limitation of TEM imaging. Rooney et al. (2017) compared microstructures using various sample preparation methods to observe the relationship between sampling methods and defect creation between layers. They applied density functional theory (DFT) calculations to determine the interlayer distance in the presence/absence of defects and compared them with the intensity profiles of real TEM cross-sectional views. The layers were more distant than the calculated results of the pristine surface, proving that defects or layer distortions can occur during sample preparation. These results indicated that sample fabrication inside a glovebox was the least damaging method of sample fabrication.

To identify the accurate sites of atoms, vacancies, or other defects, simulation and intensity profile comparison has been combined with STEM HAADF imaging for better accuracy (Krivanek et al. 2010; Pham and Yeom 2016; Bangert et al. 2017). For example, in Fig. 1, an STEM image analysis comparing a simulated image and an experimental image provides information to discern the atoms at the terminated site (Wang et al. 2017). Similarly, the type of interstitial atoms are determined using a $\mathrm{Z}$ contrast comparison with a simulated image (Bangert et al. 2017). The HAADF image that best matches the atomic configuration relaxed by the DFT revealed the structural distortion in $\mathrm{MoTe}_{2}$ from the electron irradiation effect inside the TEM (Elibol et al. 2018). Atomic bonding near the Te vacancy was also studied by investigating the shift of atomic position in the image. Zheng et al. (2019) investigated the most predominant defect formation sites in $\mathrm{WSe}_{2}$ by examining HAADF images combined with DFT calculations of the vacancy formation energies. In their research, in contrast to previous expectations, Se vacancies were preferred to $\mathrm{W}$ vacancies. Moreover, Se sites were substituted by $\mathrm{O}$ in an amorphous structure. These defect formations and interstitial observations using TEM images explicated the structural state of 2D materials, which is important for their functional use. 


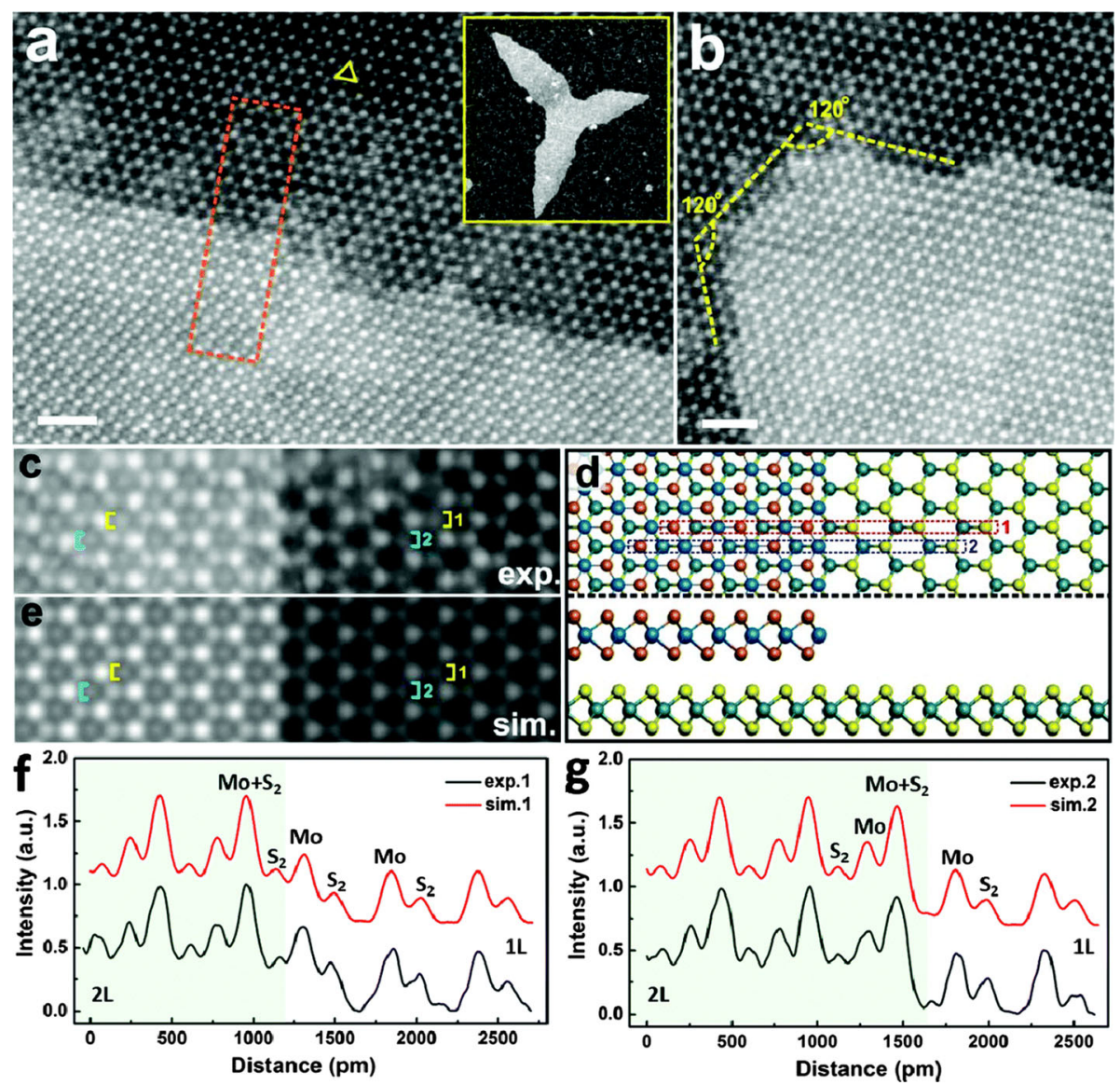

Fig. 1 ADF-STEM images showing stacked bilayer $M_{2}$. a ADF-STEM image of the tip region of stacked bilayer MoS 2 . b ADF-STEM image showing the extended bilayer $\mathrm{MoS}_{2}$ step edge. $\mathbf{c}$ Magnified image of the small region in (a). $\mathbf{d}$ Atomic model based on (c) showing both projection and side views. e Multi-slice ADF-STEM image simulation corresponding to the atomic model in (d). $\mathbf{f}$ Intensity line comparison between the simulated and experimental image of the site terminated with $S_{2}$ atoms. $\mathbf{g}$ Intensity line comparison between the simulated and experimental image of the site terminated with $\mathrm{Mo}+\mathrm{S}_{2}$ atoms. Reprinted from Wang et al. (2017) (Nanoscale 9, 13,060-13,068) with Nanoscale's permission

\section{Observation of structural distortion}

The thin layer property of 2D materials enables to induce their structural deformation in the domain scale, and this is commonly observed using TEM.

\section{$-2 D$ layer phase structural transformation}

Compared with graphene, TMD materials have few structural phases, mostly trigonal prismatic $(2 \mathrm{H})$ and octahedral $(1 \mathrm{~T}) .2 \mathrm{H}$ is in the coordination of chalcogen atom in the same place in perpendicularly situating in the different layer (Fig. 2a), with the metal between them forming the ABA-like structure, and the $1 \mathrm{~T}$ structure is in the $A B C$ stacking in which the chalcogen atoms are not in the same perpendicular line (Fig. 2b) (Manzeli et al. 2017; Gao et al. 2015). The electrical characteristics of each structure differ. $2 \mathrm{H} \mathrm{Mo-}$ and W-based materials are semiconductors, and their photon absorption gap is $1-2 \mathrm{eV}$. The $2 \mathrm{H}$ structure, distorted $1 \mathrm{~T}$ phase, and $1 \mathrm{~T}$ ' (Fig. 2c) demonstrate semi-metallic characteristics
(Huang et al. 2016; Li et al. 2016). These structures are discernable using the HAADF image (Fig. 2d and e) (Eda et al. 2012). The significance of structure-dependent electrical properties is increasing because of the changeability of the conductance in materials with the same stoichiometry. Transformations between the phases are induced by stimulating the material with electron beam irradiation. Elibol et al. (2018) reported that Te vacancies converted $1 \mathrm{H}$ structures into $1 \mathrm{~T}$ ' changes using STEM HAADF imaging and the radiation effect. The phase transformation leads to a more stable state, which minimizes the strain generated by the vacancies. The electron beam in TEM also activated inversion-domain formation and structural distortions in $\mathrm{MoS}_{2}$ (Ryu et al. 2016; Elibol et al. 2018). The $S$ vacancies induced by electron beam irradiation during atomic resolution TEM accumulated and resulted in line defects. These line defects clustered and formatted the hold in the $\mathrm{MoS}_{2}$ (Ryu et al. 2016). In $\mathrm{MoSe}_{2}$, Se vacancies induced by radiation 
a

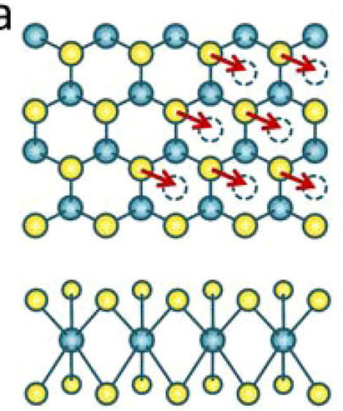

b
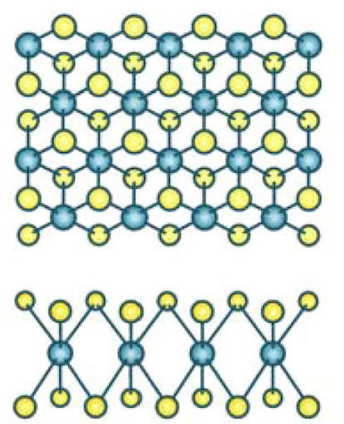

C
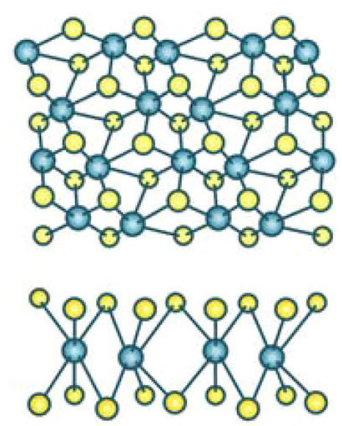

d

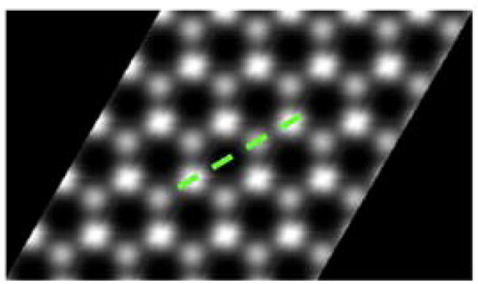

f

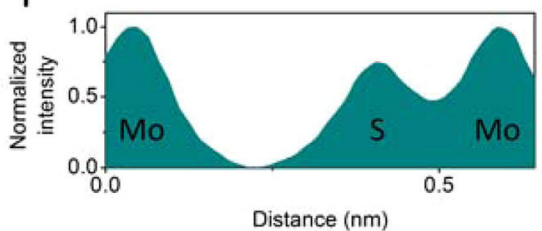

e

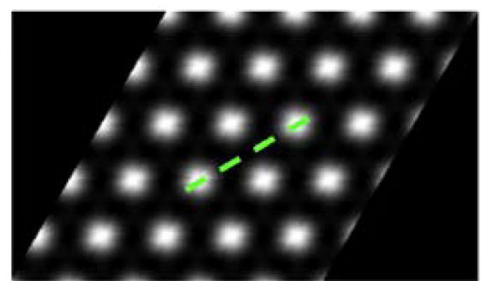

g

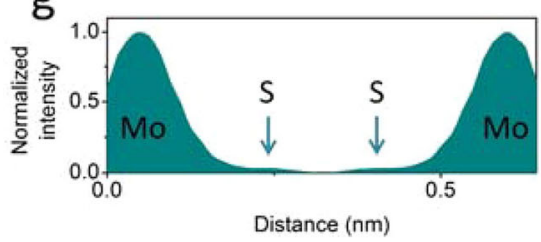

Fig. 2 (a) $2 \mathrm{H}$ (the arrows show the local S glide inducing local transformation of the $1 \mathrm{~T}$ structure), (b) $1 \mathrm{~T}$, and (c) $1 \mathrm{~T}^{\prime}$ phases viewed from the out-ofplane and in-plane axes. (d and e) Simulated HAADF STEM images of ideal (d) 2H and (e) 1 T phases. Reprinted from Eda et al. (2012) (ACS Nano 6, 73117317) with ACS Nano's permission. (f, $\mathbf{g})$ Intensity profile along the lines indicated in images $\mathbf{d}$ and $\mathbf{e}$ are shown in images $\mathbf{f}$ and $\mathbf{g}$, respectively. bulk

agglomerated into line defects and sequential atomic movement, filling the vacancies and resulting in triangular inversion symmetry. The formation mechanisms of phase changes in 2D materials have been more thoroughly understood with these studies.

\section{-periodic lattice distortion-induced modifications}

Periodic lattice distortion (PLD) has been observed in selected area electron diffraction (SAED) (Ishiguro et al. 1991; Hovden et al. 2016). PLD modulates the nuclei site and CDW to the energetically stable state (Hovden et al. 2016). Modified domains can be observed in highresolution imaging and diffraction patterns. Hovden et al. (2016) investigated the visibility of commensurate structure projection images of $1 \mathrm{~T}-\mathrm{TaS}_{2}$ PLD in 65 layers by using HAADF imaging. Without the ordering of PLD in the $\mathrm{z}$-axis direction, the superlattice could not be seen in the projection image. This visible ordering in the projection image supported the c-axis ordering of the CDW suggested in the free-energy calculations (McMillan 1975).

Moreover, the Fourier transform data of the PLD structure imaging indicates information that is not very clear in HRTEM imaging (Fig. 3) (Börner et al. 2018). The $1 \mathrm{~T}-\mathrm{TaSe}_{2}$ monolayer/graphene heterostructure CDW state pattern was obscure in the HRTEM image as shown in Fig. 3. However, in the fast Fourier transform (FFT) image, the PLD peaks are easily discerned, and the $1 \mathrm{~T}$ $\mathrm{TaSe}_{2}$ proved to be commensurate to the bulk state. This result confirmed the stability of CDW in the monolayer 1 $\mathrm{T}-\mathrm{TaSe}_{2}$ layer (Börner et al. 2018).

\section{-in situ-based structural observation}

In situ TEM experiments probe structural changes under specific conditions such as heating, electrical biasing, and cooling. The PLD/CDW observations are an example of a cryo-in situ experiment. In the experiment, the temperature was decreased until the transformation of nearly commensurate into commensurate structures occurred (Fig. 4) (Hovden et al. 2016; Börner et al. 2018; Tsen et al. 2015). In situ heating experiments on $2 \mathrm{D}$ materials can illustrate the mechanisms of structural changes according to the temperature changes. Fei et al. (2016) envisaged the structural transformation mechanisms from precursor to vertical $\mathrm{MoS}_{2}$ layers by using a heating holder. Moreover, the structural orientation changes from vertical to horizontal were identified by HRTEM and SAED imaging. 


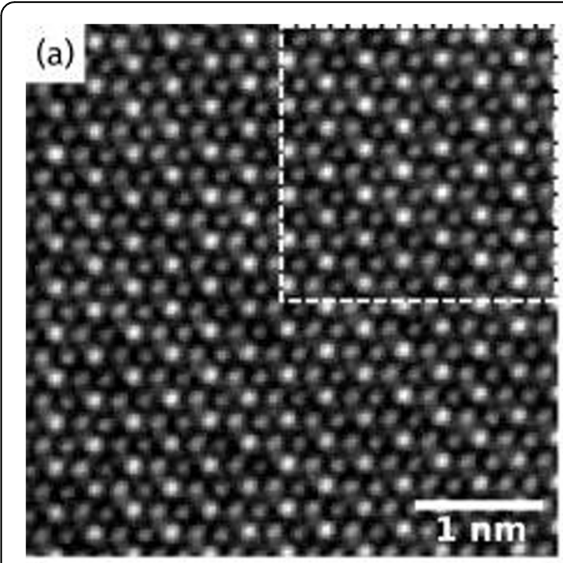

\section{(b)}
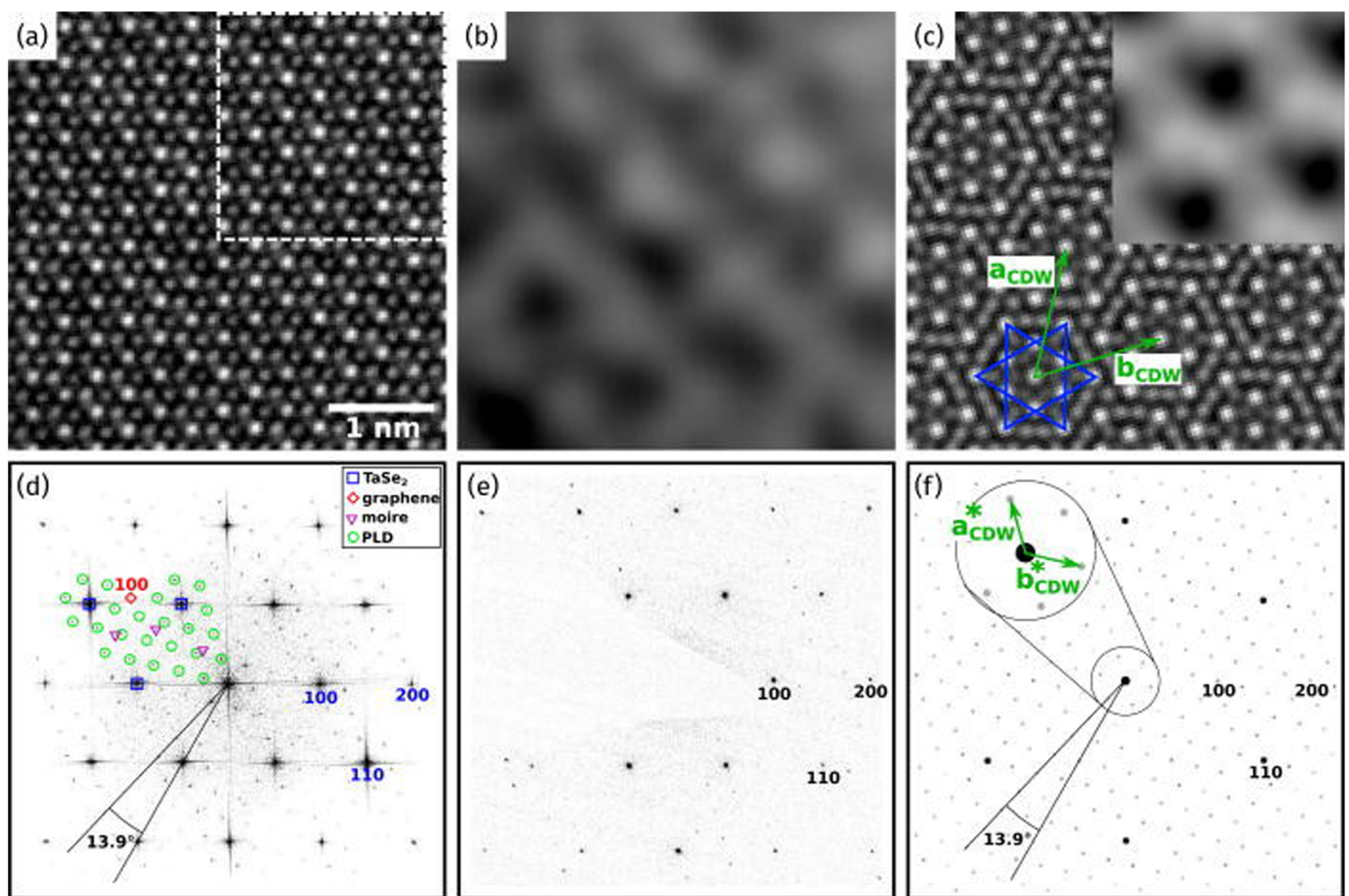

Fig. 3 a Experimental AC-HRTEM 1 T-TaSe2/graphene heterostructure at $80 \mathrm{kV}$. b Contrast variation due to the CCDW/PLD. c Simulated ACHRTEM image of the CCDW/PLD in a single 1 T-TaSe2 layer. The Star of David structure is marked in blue. $\mathbf{d}$ The Fourier transformation of image (a) showing the PLD structure peaks. e Visible satellite spots of the CCDW/PLD. f Simulated kinematic electron diffraction pattern of the CCDW/ PLD in a single 1 T-TaSe2 layer. Reprinted from Börner et al. (2018) (Appl. Phys. Lett. 113, 173,103) with Applied Physics Letters' permission
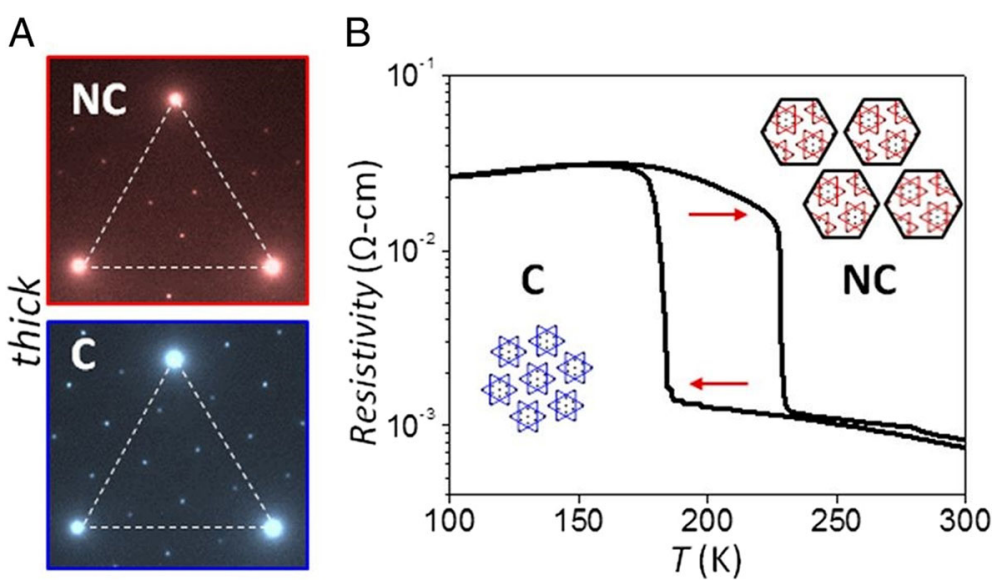

Fig. 4 Nearly commensurate to commensurate phase transition in 1 T-TaS2 and CDW suppression by oxidation in thin flakes. a TEM diffraction images of 1 T-TaS2 at $295 \mathrm{~K}$ (red, NC phase) and $100 \mathrm{~K}$ (blue, c phase). Weaker peaks are due to CDW distortion. b Resistivity vs. temperature of bulk 1 T-TaS2 crystal around the first-order, NC-C transition. Reprinted from Tsen et al. (2015) (Proc. Natl. Acad. Sci. 112, 15,054-15,059) with National Academy of Sciences of Prospects of the United States of America's permission 


\section{Observation of the microstructure of 2D materials in combination with electric measurements}

The alteration in the twist angle between the layers in a van der Waals structure is applied for electronic characteristic tuning in 2D materials (Ju et al. 2017; Cao et al. 2018; Ke et al. 2019; Yoo et al. 2019). The bilayer twist method in graphene shows charge carrier density tunability with the precisely adjusted interlayer angle ( $\mathrm{Li}$ et al. 2010; Kim et al. 2016; Cao et al. 2018). Recently, a 2D superlattice structure, called the magic angle $\left(1.1^{\circ}\right)$ twisted bilayer graphene (MA-TBG), showed a flat band near zero Fermi energy, and the insulating property was revealed (Cao et al. 2018). Insulating states resulted from the competition of Coulomb energy and quantum-like energy and were consistent with a Mott-like insulating state. This state can be changed into a superconducting state with electron doping and at temperatures reaching $1.7 \mathrm{~K}$ (Cao et al. 2018). Moreover, the TBG superlattice showed a quasi-periodic moiré pattern, and the moiré potential trapped the interlayer excitons, which functioned as the charge carriers (Woods et al. 2014; Seyler et al. 2019).

Yoo et al. (2019) showed the atomic and electronic reconstruction at the van der Waals interface in twisted bilayer graphene. Twisted graphene bilayers changed from an incommensurate domain to a strong commensurate domain with the gradual change in the angle (Yoo et al. 2019). Similar phenomena were reported in different $2 \mathrm{D}$ van der Waals bilayers and proved by scanning probe microscopy and scanning tunneling microscopy, and the results showed surface reconstruction at the interface (Woods et al. 2014). Yoo et al. (2019) obtained the TEM dark-field image and diffraction pattern and compared the experimental result with the simulations in both reconstructed and unreconstructed states. The satellite peaks shown in the experiments were not observed in the unconstructed surface, which proves the atomic reconstruction in twisted bilayer graphene. In the commensurate state at the magic angle of $1.1^{\circ}$, the calculation of the unreconstructed state interlayer band structure of the twisted bilayer graphene did not comply with the real data. The experimental data showed better consistency with the reconstruction band structure. Moreover, the traverse displacement field applied in the moire patterns proved the 1D channel formation due to atomic reconstruction (Yoo et al. 2019). These experimental results demonstrated surface reconstruction between the bilayers.

In PLD, the commensurate and incommensurate structures are also related to the electrical resistivity; therefore, microstructural analysis combined with the electric measurements was conducted. For the functional use of $1 \mathrm{~T}-\mathrm{TaS}_{2}$, a persisting and nearly commensurate structure throughout the temperature changes is necessary for better electric device conductivity. Tsen et al. (2015) measured the resistivity of $1 \mathrm{~T}-\mathrm{TaS}_{2}$ of different thicknesses with changes in temperature. The resistivity-temperature curve of the thin sample showed the persistence of the nearly commensurate state. TEM diffraction peaks from 2 $\mathrm{nm}$ and $12 \mathrm{~nm}$ layers were compared and demonstrated the disappearance of commensurate peaks in the $2 \mathrm{~nm}$ sample. This result was consistent with the electronic measurements (Tsen et al. 2015). These experiments proved the importance of TEM imaging and diffraction in correlation with electrical property measurements to better understand the origin of material properties on an atomic scale.

\section{Conclusions}

Because the electronic and optical properties of 2D materials can be tuned with structural changes, previous investigations focused on microstructural analyses. TEM studies on 2D materials demonstrated the defects, interstitial atomic formations, and other structural scale distortions and atomic configurations. Beginning with lowvoltage electron beams in which the $2 \mathrm{D}$ material sample remains stable, the progress of research has revealed many mechanisms in the formation of structural changes. Recent microstructural studies have assessed the structural distortion-induced electrical properties. These studies combined TEM analyses with other electrical measurements, e.g., conductivity measured using applied traverse field voltage and resistivity measured by voltage changes. These results have been supported by prior studies on 2D material electronic coupling and other unique electronic characteristics induced by microstructural changes.

Although progress has been made in this area, 2D material research continues to advance. Combined with technical developments in TEM, which have led to subAngstrom resolution analysis in low-voltage TEM, 2D material research will become a better understood field with the potential for exciting discoveries.

\section{Abbreviations \\ 2D materials: Two-dimensional materials; CDW: Charge density wave; FFT: Fast Fourier transform; HAADF: High-angle annular dark field; HRTEM: High-resolution transmission electron microscopy; MA: Magic angle $\left(1.1^{\circ}\right)$; PLD: Periodic lattice distortion; SAED: Selected area electron diffraction; STEM: Scanning transmission electron microscopy; TBG: Twisted bilayer graphene; TEM: Transmission electron microscopy; TMD: Transition metal dichalcogenides}

\section{Acknowledgements}

Not applicable.

\section{Authors' contributions}

YYC performed data collection and analysis and wrote the entire manuscript. MK and $\mathrm{HNH}$ supervised the entire study process, including analysis. All authors read and approved the final manuscript.

\section{Funding}

This work was supported by the National Research Foundation of Korea (NRF) grants funded by the Ministry of Science and ICT (MSIT) [No. 2015R1A5A1037627]. 


\section{Availability of data and materials}

Not applicable.

\section{Competing interests}

The authors declare that they have no competing interests.

Received: 23 August 2019 Accepted: 10 October 2019

\section{Published online: 04 November 2019}

\section{References}

U. Bangert, A. Stewart, E. O'Connell, E. Courtney, Q. Ramasse, D. Kepaptsoglou, H. Hofsäss, J. Amani, J.S. Tu, B. Kardynal, lon-beam modification of 2-D materials-Single implant atom analysis via annular dark-field electron microscopy. Ultramicroscopy 176, 31-36 (2017)

P.C. Börner, M.K. Kinyanjui, T. Björkman, T. Lehnert, A.V. Krasheninnikov, U. Kaiser, Observation of charge density waves in free-standing 1T-TaSe2 monolayers by transmission electron microscopy. Appl. Phys. Lett. 113, 173103 (2018)

M. Calandra, 2D materials: Charge density waves go nano. Nat. Nanotechnol. 10, 737-738 (2015)

Y. Cao, V. Fatemi, S. Fang, K. Watanabe, T. Taniguchi, E. Kaxiras, P. Jarillo-Herrero, Unconventional superconductivity in magic-angle graphene superlattices. Nature 556, 43-50 (2018)

H. Castro Neto, Charge density wave, superconductivity, and anomalous metallic behavior in 2D transition metal dichalcogenides. Phys. Rev. Lett. 86, 43824385 (2001)

W. Chen, J. Choe, E. Morosan, Charge density waves in strongly correlated electron systems. Rep. Prog. Phys. 79, 084505 (2016)

G. Eda, T. Fujita, H. Yamaguchi, D. Voiry, M. Chen, M. Chhowalla, Coherent atomic and electronic heterostructures of single-layer MoS 2. ACS Nano 6, 73117317 (2012)

K. Elibol, T. Susi, G. Argentero, M.R.A. Monazam, T.J. Pennycook, J.C. Meyer, J. Kotakoski, Atomic structure of intrinsic and electron-irradiation-induced defects in MoTe2. Chem. Mater 30, 1230-1238 (2018)

L. Fei, S. Lei, W.B. Zhang, W. Lu, Z. Lin, C.H. Lam, Y. Chai, Y. Wang, Direct TEM observations of growth mechanisms of two-dimensional MoS 2 flakes. Nat. Commun. 7, 1-7 (2016)

G. Gao, Y. Jiao, F. Ma, Y. Jiao, E. Waclawik, A. Du, Charge mediated semiconducting-to-metallic phase transition in molybdenum disulfide monolayer and hydrogen evolution reaction in new 1T' phase. J. Phys. Chem C 119, 13124-13128 (2015)

J.H. Garcia, M. Vila, A.W. Cummings, S. Roche, Spin transport in graphene/transition metal dichalcogenide heterostructures. Chem. Soc. Rev. 47, 3359-3379 (2018)

J. He, K. Hummer, C. Franchini, Stacking effects on the electronic and optical properties of bilayer transition metal dichalcogenides MoS 2, MoSe 2, WS 2 and WSe 2. Phys. Rev. B-Condens. Matter Mater. Phys. 89, 1-11 (2014)

M. Hossain, Z. Zhao, W. Wen, X. Wang, J. Wu, L. Xie, Recent advances in twodimensional materials with charge density waves: Synthesis, characterization and applications. Crystals 7, 298 (2017)

R. Hovden, A.W. Tsen, P. Liu, B.H. Savitzky, I. El Baggari, Y. Liu, W. Lu, Y. Sun, P. Kim, A.N. Pasupathy, L.F. Kourkoutis, Atomic lattice disorder in chargedensity-wave phases of exfoliated dichalcogenides (1T-TaS 2). Proc. Natl. Acad. Sci. 113, 11420-11424 (2016)

H.H. Huang, X. Fan, D.J. Singh, H. Chen, Q. Jiang, W.T. Zheng, Controlling phase transition for single-layer MTe2 ( $\mathrm{M}=\mathrm{Mo}$ and $\mathrm{W})$ : Modulation of the potentia barrier under strain. Phys. Chem. Chem. Phys. 18, 4086-4094 (2016)

T. Ishiguro, H. Sato, Electron microscopy of phase transformation in 1T-TaS2. Phys Rev. B 44, 2046-2060 (1991)

M.D. Johannes, I.I. Mazin, Fermi surface nesting and the origin of charge density waves in metals. Phys. Rev. B-Condens. Matter Mater. Phys. 77, 1-9 (2008)

L. Ju, W. Lei, C. Ting, T. Takashi, W. Kenji, L. Steven, R. Farhan, P. Jiwoong, H. James, W. Feng, M. Paul, Tunable excitons in bilayer graphene. Science 358 $1-4(2017)$

Q. Ke, X. Zhang, W. Zang, A.M. Elshahawy, Y. Hu, Q. He, S.J. Pennycook, Y. Cai, J. Wang, Strong charge transfer at 2H-1T phase boundary of MoS2 for superb high-performance energy storage. Small 15, 1-11 (2019)

K. Kim, M. Yankowitz, B. Fallahazad, S. Kang, H.C.P. Movva, S. Huang, S. Larentis, C. M. Corbet, T. Taniguchi, K. Watanabe, S.K. Banerjee, B.J. Leroy, E. Tutuc, Van der Waals heterostructures with high accuracy rotational alignment. Nano Lett. 16, 1989-1995 (2016)

O.L. Krivanek, M.F. Chisholm, V. Nicolosi, T.J. Pennycook, G.J. Corbin, N. Dellby, M. F. Murfitt, C.S. Own, Z.S. Szilagyi, M.P. Oxley, S.T. Pantelides, S.J. Pennycook,
Atom-by-atom structural and chemical analysis by annular dark-field electron microscopy. Nature 464, 571-574 (2010)

G. Li, A. Luican, J.M.B. Lopes Dos Santos, A.H. Castro Neto, A. Reina, J. Kong, E.Y. Andrei, Observation of Van Hove singularities in twisted graphene layers. Nat. Phys. 6, 109-113 (2010)

Y. Li, K.A.N. Duerloo, K. Wauson, E.J. Reed, Structural semiconductor-to-semimetal phase transition in two-dimensional materials induced by electrostatic gating. Nat. Commun. 7, 1-8 (2016)

Z. Lin, B.R. Carvalho, E. Kahn, R. Lv, R. Rao, H. Terrones, M.A. Pimenta, M. Terrones, Defect engineering of two-dimensional transition metal dichalcogenides. 2D Mater. 3, 022002 (2016)

S. Manzeli, D. Ovchinnikov, D. Pasquier, O. Yazyev, A. Kis, 2D transition metal dichalcogenides. Nat. Rev. Mater. 2, 1-15 (2017)

W.L. McMillan, Landau theory of CDW in transition-metal dichalcogenides. Phys. Rev. B 12, 1187-1196 (1975)

J.C. Meyer, F. Eder, S. Kurasch, V. Skakalova, J. Kotakoski, H.J. Park, S. Roth, A. Chuvilin, S. Eyhusen, G. Benner, A.V. Krasheninnikov, U. Kaiser, Accurate measurement of electron beam induced displacement cross sections for single-layer graphene. Phys. Rev. Lett. 108, 1-6 (2012)

V.P. Pham, G.Y. Yeom, Recent advances in doping of molybdenum disulfide: Industrial applications and future prospects. Adv. Mater. 28, 9024-9059 (2016)

R. Ribeiro-Palau, C. Zhang, K. Watanabe, T. Taniguchi, J. Hone, C. Dean, Twistable electronics with dynamically rotatable heterostructures. Science $361,690-$ 693 (2018)

P. Rivera, J.R. Schaibley, A.M. Jones, J.S. Ross, S. Wu, G. Aivazian, P. Klement, K. Seyler, G. Clark, N.J. Ghimire, J. Yan, D.G. Mandrus, W. Yao, X. Xu, Observation of long-lived interlayer excitons in monolayer MoSe 2-WSe 2 heterostructures. Nat. Commun. 6, 4-9 (2015)

P. Rooney, A. Kozikov, A.N. Rudenko, E. Prestat, M.J. Hamer, F. Withers, Y. Cao, K.S. Novoselov, M.I. Katsnelson, R. Gorbachev, S.J. Haigh, Observing imperfection in atomic interfaces for van der Waals heterostructures. Nano Lett. 17, 52225228 (2017)

G. Ryu, J. Lee, N. Kim, Y. Lee, Y. Kim, M. Kim, C. Lee, Z. Lee, Line-defect mediated formation of hole and Mo clusters in monolayer molybdenum disulfide. $2 \mathrm{D}$ Mater 3, 014002 (2016)

K.L. Seyler, P. Rivera, H. Yu, N.P. Wilson, E.L. Ray, D.G. Mandrus, J. Yan, W. Yao, X. $\mathrm{Xu}$, Signatures of moiré-trapped valley excitons in MoSe 2 WSe 2 heterobilayers. Nature 567, 66-70 (2019)

W. Tsen, R. Hovden, D. Wang, Y.D. Kim, J. Okamoto, K.A. Spoth, Y. Liu, W. Lu, Y. Sun, J.C. Hone, L.F. Kourkoutis, P. Kim, A.N. Pasupathy, Structure and control of charge density waves in two-dimensional 1T-TaS 2. Proc. Natl. Acad. Sci. $112,15054-15059$ (2015)

H. Wang, H. Yuan, S. Sae Hong, Y. Li, Y. Cui, Physical and chemical tuning of two-dimensional transition metal dichalcogenides. Chem. Soc. Rev. 44, 2664-2680 (2015)

Q.H. Wang, K. Kalantar-Zadeh, A. Kis, J.N. Coleman, M.S. Strano, Electronics and optoelectronics of two-dimensional transition metal dichalcogenides. Nat. Nanotechnol. 7, 699-712 (2012)

S. Wang, H. Sawada, C.S. Allen, A.I. Kirkland, J.H. Warner, Orientation dependent interlayer stacking structure in bilayer MoS2 domains. Nanoscale 9, 1306013068 (2017)

C.R. Woods, L. Britnell, A. Eckmann, R.S. Ma, J.C. Lu, H.M. Guo, X. Lin, G.L. Yu, Y. Cao, R.V. Gorbachev, A.V. Kretinin, J. Park, L.A. Ponomarenko, M.I. Katsnelson, Y.N. Gornostyrev, K. Watanabe, T. Taniguchi, C. Casiraghi, H.J. Gao, A.K. Geim, K.S. Novoselov, Commensurate-incommensurate transition in graphene on hexagonal boron nitride. Nat. Phys. 10, 451-456 (2014)

J. Yan, J. Xia, X. Wang, L. Liu, J.L. Kuo, B.K. Tay, S. Chen, W. Zhou, Z. Liu, Z.X. Shen, Stacking-dependent interlayer coupling in trilayer MoS2 with broken inversion symmetry. Nano Lett. 15, 8155-8161 (2015)

H. Yoo, R. Engelke, S. Carr, S. Fang, K. Zhang, P. Cazeaux, S.H. Sung, R. Hovden, A. W. Tsen, T. Taniguchi, K. Watanabe, G.C. Yi, M. Kim, M. Luskin, E.B. Tadmor, E. Kaxiras, P. Kim, Atomic and electronic reconstruction at the van der Waals interface in twisted bilayer graphene. Nat. Mater. 18, 448-453 (2019)

Y.J. Zheng, Y. Chen, Y.L. Huang, P.K. Gogoi, M.Y. Li, L.J. Li, P.E. Trevisanutto, Q. Wang, S.J. Pennycook, A.T.S. Wee, S.Y. Quek, Point defects and localized excitons in 2D WSe2. ACS Nano 13, 6050-6059 (2019)

\section{Publisher's Note}

Springer Nature remains neutral with regard to jurisdictional claims in published maps and institutional affiliations. 